\title{
Pattern Classification using Three Axis Accelerometer and Magnetic Sensor Toothbrush
}

\author{
S Prasath Alias Surendhar S., G.Bupeshb, Sowmiya. R, Abinaya. K.
}

\begin{abstract}
The idea of savvy toothbrush, fit for observing brushing movement, introduction through the hold pivot, amid tooth brushing was recommended in our past investigation. In this investigation, we depict a tooth brushing design order calculation utilizing three-pivot accelerometer and three-hub attractive sensor. We have discovered that wrong tooth brushing design indicated explicit moving examples. So as to follow the position and introduction of toothbrush in a mouth, we have to know outright organize data of toothbrush. By applying tilt-repaid azimuth (heading) figuring calculation, which is commonly utilized in little telemetries gadgets, we could discover the tendency and introduction data of toothbrush. To evaluate the plausibility of the proposed calculation, 8 brushing examples were performed by 6 individual solid subjects. The proposed calculation demonstrated the discovery proportion of $98 \%$. This investigation demonstrated that the proposed checking framework was imagined to help dental consideration faculty in patient training and guidance in oral cleanliness with respect to brushing style.
\end{abstract}

Index Terms: Accelerometer, Orientation, Telemetries, Plausibility, Pivot, Azimuth.

\section{INTRODUCTION}

Parkinson's malady is a dynamic sensory system issue that influences development. Side effects begin steadily, in some cases beginning with a scarcely recognizable tremor in only one hand. Tremors are normal, however the turmoil likewise generally causes solidness or moderating of development. In the beginning times of Parkinson's malady, your face may demonstrate next to zero appearance. Your arms may not swing when you walk. Your discourse may turn out to be delicate or slurred. [1] Parkinson's malady side effects decline as your condition advances after some time. Despite the fact that Parkinson's illness can't be relieved, drugs may altogether improve your side effects. Incidentally, your specialist may recommend medical procedure to direct certain locales of your mind and improve your side effects. Parkinson's infection signs and indications can be diverse for everyone.[2] Early signs might be mellow and go unnoticed. Side effects frequently start

Revised Manuscript Received on July 22, 2019.

Prasath Surendhar S., Assistant Professor, Department Of Biomedical Engineering, BIHER, Chennai 600073, India

Bupeshb G. Associate Professor/ Director,Faculty of Medical Biochemistry \& Research Development Wing, Sree Balaji Medical College Hospital, BIHER, Chennai 6000044, India

Sowmiya R., Research Scholar, Department Of Biomedical Engineering, BIHER, Chennai 600073, India.

Abinaya K. Research Scholar, Department Of Biomedical Engineering, BIHER, Chennai 600073, India on one side of your body and for the most part stay more terrible on that side, even after manifestations start to influence the two sides. Parkinson's signs and indications may incorporate, for example, tremor, impeded development, inflexible muscles, and discourse changes, composing changes, weakened stance and parity, loss of programmed development. [3] In Parkinson's ailment, certain nerve cells (neurons) in the cerebrum slowly separate or bite the dust. A considerable lot of the manifestations are because of lost neurons that produce a substance detachment in your mind called dopamine. At the point when dopamine levels decline, it causes unusual mind movement, prompting indications of Parkinson's illness. [4] The reason for Parkinson's malady is obscure, yet a few elements seem to assume a job, including: qualities, ecological triggers. Specialists have likewise noticed that numerous progressions happen in the cerebrums of individuals with Parkinson's sickness, despite the fact that it's not clear why these progressions happen. These progressions include: the nearness of lewy bodies, Alpha-synuclein is found inside lewy bodies. [5] Risk factors for Parkinson's malady include: Age, Heredity, sex, presentation to poisons. Parkinson's illness is regularly joined by these extra issues, which might be treatable we may encounter psychological issues (dementia) and thinking difficulties.[6] These typically happen in the later phases of Parkinson's infection. We may encounter gloom, here and there in the in all respects beginning periods. Getting treatment for wretchedness can make it simpler to hand the different difficulties of Parkinson's disease [7].

\section{MATERIALS AND METHOD}

The MPU6050 is a 6 DOF (degrees of opportunity) or a six-pivot IMU sensor, which implies that it gives six qualities as yield: three qualities from the accelerometer and three from the spinner. The MPU6050 is a sensor dependent on MEMS (smaller scale electro mechanical frameworks) innovation. MPU6050 sensor module is finished 6-hub movement GPS beacon. It joins 3-pivot whirligig, 3-hub accelerometer and advanced movement processor all in little bundle [8] .Also, it has extra component of on - chip temperature sensor. It has 12C transport interface to speak with the microcontroller. This gadget intended for the low power, ease and superior necessities of advanced mobile phones, tablets and wearable sensors [9]. It fuses Invent sense's movement combination and run time adjustment firm wave that empowers fabricates to dispose of the expensive and complex choice, capability and framework level mix of discrete gadgets in movement empower items, that sensor combination calculations and

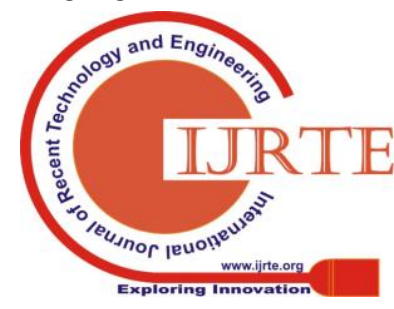


alignment makers convey ideal execution for customers [10].

A gadget comprising of a wheel or circle mounted so $t$ can turn quickly around a pivot which is without itself to modify in bearing [11]. The introduction of the pivot isn't influenced by tilting of the mounting, so gyroscopes can be utilized to give strength or keep up a reference heading in route frameworks, programmed pilots and stabilizers. At the point when things pivot around a hub they have what's called rakish speed. A turning wheel can be estimated in cycles every second (RPS) or degree every second. The run of the mill two-pivot accelerometer provides clients a guidance of gravity in a flying machine, advanced mobile phone, vehicle or other gadget. In correlation, a whirligig is proposed to decide a rakish position dependent on the standard of Inflexibility of room [11-13].

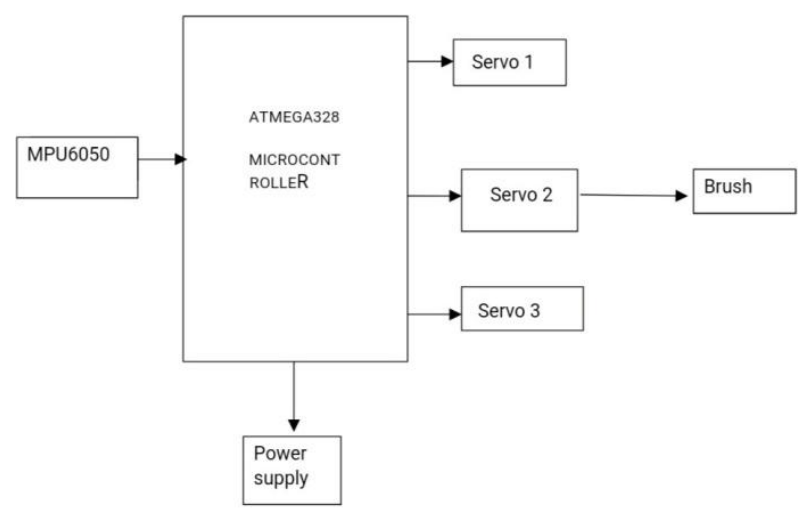

Figure a: Block Diagram for Magnetic sensor toothbrush

Atmega 328 AVR is microcontroller family, the ATmega8. This part has numerous highlights like that of ATmega32. Be that as it may, it has diminished number of highlights and abilities, yet it has enough highlights to work with. Presently given me a chance to reveal to you that in the event that you need to accumulate learning and in the meantime need to do it in less expense than the financial plan of ATmega32, you can consider making ventures with ATmega8. All things considered, one element you won't probably acknowledge is the JTAG interface. In any case, rest of the highlights. The magnetic sensor is the input signal which is known as MPU6050 sensor. This sensor is made up of MEMS. This magnetic sensor is connected to the power supply ATMEGA328. From that microcontroller three servo motors are arrange in same line for connection and it give pulse width module that method is known as step pulse method [14]. By using with the sensor the three servomotor rotate at a different angle. The toothbrush model will rotate with three axis like acute angle, right angle and obtuse angle. And it gives three outputs in $\mathrm{X}, \mathrm{Y}, \mathrm{Z}$ directions of rotation [15-17].

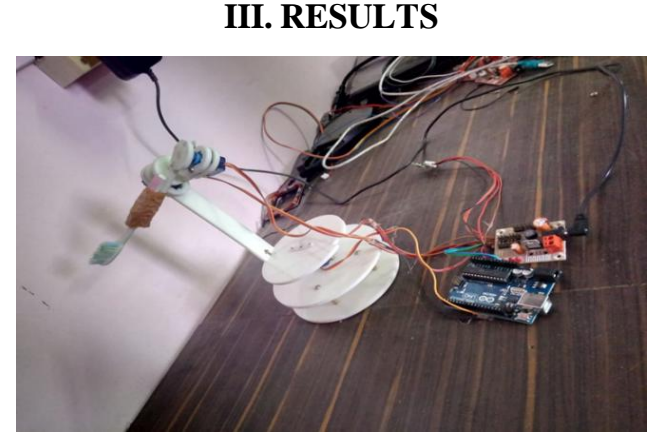

Figure b: $45^{\circ}$ angle rotation

In the above fig. 2 shown that toothbrush rotate at the angle of $45^{\circ}$ by the input signal given by the magnetic sensor. This magnetic sensor gives more rotation as per the patient comfort.

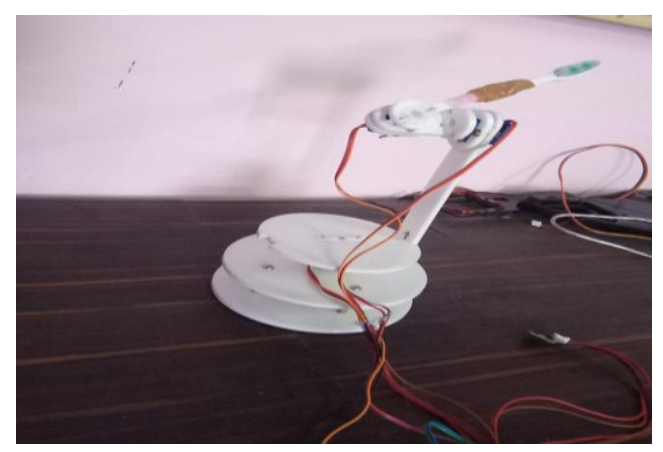

Figure c: $60^{\circ}$ angle rotation

In the above fig. 3 shown that toothbrush rotate at the angle of $60^{\circ}$ by the input signal given by the magnetic sensor. This magnetic sensor gives more rotation as per the patient comfort.

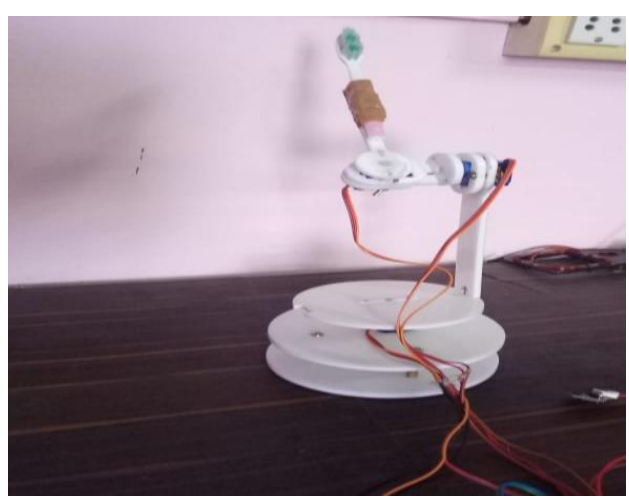

Figure d: $90^{\circ}$ angle rotation

In the above fig. 4 shown that toothbrush rotate at the angle of $90^{\circ}$ by the input signal given by the magnetic sensor. This magnetic sensor gives more rotation as per the patient comfort

\section{IV.CONCLUSION}

The smart toothbrush technique for applying the medical field to give the solution for the patients those who are all affected by the paralysis disease. This tooth brush is compactable and gives protection to the jaws. And cost effective when compared with existing researches and also this tooth gives $100 \%$ efficiency for 
the normal and children's. In this undertaking, savvy tooth brush serves to access the teeth gums and all blood supply and nerve supply in a delicate way. Contrasted with electrical tooth brush this example grouping of tooth brush by utilizing attractive sensor demonstrates the great outcome through investigations.

\section{V.DISCUSSION}

From the past and this investigation, we tried plausibility of tooth brushing observing and brushing design arrangement calculation plans. In any case, there are as yet numerous issues and issues to be illuminated. Particularly, when brushing inside dental curves, we have still cloud tendency data whether outside upper curve or not.

Likewise, we have to segregate the course of toothbrush in the case of brushing left/right side or front side. However, we expectate that on the off chance that we could accurately appraise the heading data, which isn't utilized in this examination despite the fact that we determined from estimated attractive sensors, previously mentioned issues could be tackled effectively. We are presently intending to do clinical examination in dental center (preventive dentistry) for field trial of savvy toothbrush and order.

\section{REFERENCES}

1. H Huang, S Lin, "Tooth brushing recognition using neural networks" pp 309-310, 2017

2. Z Quyang, J hu, J Niu, “An assymmetrical acoustic field detection system for dialy tooth brushing monitoring", pp. 1-6, 2017

3. H-S Lee, H-I Jung, S-M Kang, H-E Kim , "Evaluation of bristle end rounding pattern of children tooth brushes using scanning electron microscopy and stero microscopy" vol. 15, no.2, pp.120-127, 2017

4. Macro Macron, Augesto sarli, Stefeno tubaro, " Smart Tooth brushing: inertial measurement sensors fusion with visual tracking", European conference on computer vision, pp.484-494, 2016.

5. J.Korpela, R Miyaji, T Maekawa, K Nozaki, “ Toothbrushing performance evalution using,smart phone audio based on hybrid hmm recognition SVM regression model " , Journal of information processing, vol.24,no.2, pp.302-313,2016.

6. H Huang, S Lin, " Toothbrushing monitoring using wrist watch" ,ACM conference on embedded network sensor system CD-ROM, pp. 202-215,2016.

7. J.Korpela, R Miyaji , T Maekawa, K Nozaki, "Evaluating toothbrush performance with smart phone sound data",ACM International joint conference on pervasive and Ubiquitous computing, pp.109-120,2015.

8. I Saitoh, Y Yu ,D Tomiyama, "User evaluation of tooth brush and arm joint motion during tooth brushing ", clinical oral investigations , vol.19,no.6,pp.1451-1462,2015.

9. KD Kim, JS jeong, HN lee, Y Gu, KS Kim, “ Efficacy of computer assisted, 3D motion capture tooth brushing instruction",clinical oral investigations, vol.19, no.6,pp 1389-1394,2015.

10. K.Kabayashi, T ohtsuki, "User identification of based on tooth brushing information using three axis accelerometer", International joint conference on pervasive and ubiquitous computing and proceedings of the 2015 ACM International symposium on wearble, 2015.

11. Wolff, C. Springer, " Toothbrushing education via a smart software visualization system",Journal of periodontology ,vol.84,no.2,pp. $186-195,2013$

12. Jomg-Jae, lee,fil-jae lee, Kyeong seop kim, wonse parle,kee-Deog Dosik Hwang, "Tooth brushing region detection of using three-axis accelerometer \&magnetic sensor", IEEE Transcations of biomedical ,vol.59,no.3,pp.872-881,2012.

13. Juha - pekka, purunen,Ari visa, Jussi callin ,Jouni Kantala, Nan Avdoveski, “ Intelligent toothbrushing monitoring devices",US-Patent 8,vol.337,no.213,2012.

14. Young-jae lee,pil-jae lee, jeong -soap kim, jin-sun,jeong wonse,"Quantitative assessment of tooth brushing education efficacy using smart toothbrush ",International conference on Biomedical Engineering and Informatics (BMEI) 2, pp.1160-1164,2011.

15. JN lee,KH lee, KS Kim, DJ Kim, "Development of smart toothbrushing monitoring system for ubiquitous healthcare",Proceeding of the 28 th IEEE EMBS Annual conference, newyork,pp.6422-6425,2006.
16. S.Y .Cho and C.G park, "A Calibration Technique for a Two- Axis magnetic compass in telematics Devices",ETRI Journal , vol27,no.3,pp.280-288,june 2005.

17. S.Y. Cho and C.G. Park, "tilt compensation Algorithm for 2-Axis magnetic compass”, IEEE Electronics Lilt .,vol.39,no. 22, oct 2003.

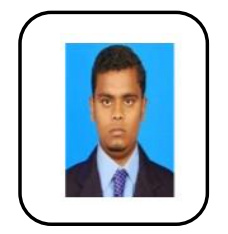

\section{AUTHORS PROFILE}

Prasath Surendhar S., was born on 1989 in Chennai. M.Tech from Department of Biomedical Engineering, VIT University in 2012. He worked as Assistant Professor Biomedical Department -Vel Tech Multitech Engineering College - Chennai from 2013 to 2014 . He currently is working Assistant Professor - Biomedical Department-BIHER, Chennai

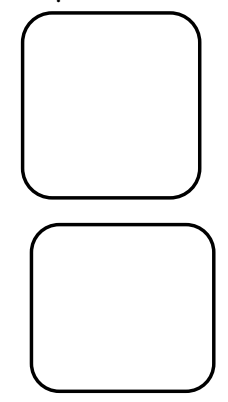

Bupeshb G was born on 1980 in Chennai, Completed Ph.D in Biochemistry in 2011 Associate Professor/ Director,Faculty of Medical Biochemistry \& Research Development Wing, Sree Balaji Medical College Hospital, BIHER,

Sowmiya $\mathbf{R}$ was born on 1996 in Tamilnadu. She is great interest towards medical field, she opt to take graduation in (B.Tech) Biomedical Engineering from University of BIHER and doing research activities.

Abinaya K was born on 1996 in Tamilnadu. She is great interest towards medical field, she opt to take graduation in (B.Tech) Biomedical Engineering from University of BIHER and doing research activities. 